\title{
Aspects of Operation of the Fermilab Booster RF System at Very High Intensity
}

\author{
J.E. Griffin \\ Fermi National Accelerator Laboratory \\ P.O. Box 500, Batavia, Illinois 60510
}

April 1996 


\section{Disclaimer}

This report was prepared as an account of work sponsored by an agency of the United States Government. Neither the United States Government nor any agency thereof, nor any of their employees, makes any warranty, express or implied, or assumes any legal liability or responsibility for the accuracy, completeness, or usefulness of any information, apparatus, product, or process disclosed, or represents that its use would not infringe privately owned rights. Reference herein to any specific commercial product, process, or service by trade name, trademark, manufacturer, or otherwise, does not necessarily constitute or imply its endorsement, recommendation, or favoring by the United States Government or any agency thereof. The views and opinions of authors expressed herein do not necessarily state or reflect those of the United States Government or any agency thereof. 
J.E. Griffin

\section{ASPECTS OF OPERATION OF THE FERMILAB BOOSTER RF SYSTEM AT VERY HIGH INTENSITY}

\section{INTRODUCTION}

The purpose of this note is to examine the likelihood and problems associated with operation of the Fermilab Booster if system as it presently exists, or with only minor modifications, at beam intensity approaching $5 \times 10^{13}$ protons per pulse. Beam loading of the rf system at such an intensity will be one order of magnitude larger than at the present operation level. It is assumed that the injection energy will be raised to $1 \mathrm{GeV}$ with no major increase in the injected energy spread (longitudinal emittance). The beam will be bunched by adiabatic capture as is presently done although it may be necessary to remove one or two bunches prior to acceleration to allow clean extraction at $8 \mathrm{Gev}$.

At very high intensity the charge in each bunch will interact with the vacuum chamber impedance (and with itself) in such a way as to reduce in some cases the bucket area generated by the If voltage ${ }^{[1]}$. Because this decrement must be made up by changes in the $\mathrm{rf}$ ring voltage if the required bucket area is to be maintained, these effects must be taken into consideration in any analysis of the capability of the rf system to accelerate very large intensity.

\section{FUNDAMENTAL BEAM LOADING}

It is common to represent individual if cavities near resonance by a lumped $\mathrm{RLC}$ circuit ${ }^{[3]}$. Fig. 1a shows such a representation with the power source represented explicitly by a current source in parallel with a source resistance, which may be the anode resistance of a tetrode power amplifier tube in this case. In Fig. $1 \mathrm{~b}$ the generator current has been transformed (downward) by the voltage step-up ratio and applied directly to the gap. The source resistance has been increased by the square of the ratio and placed in parallel with the cavity unloaded shunt resistance creating an effective $R_{\text {sh }}$. The resistance labelled $Z_{\mathrm{o}}(\omega)$ in series with the capacitor reflects the fact that the impedance presented to the beam at very high frequency does not approach zero, but reaches a small varying value near the cavity characteristic impedance. These impedances contribute to the ring impedance and to the beam-vacuum chamber interaction at high frequency.

Each cavity is characterized by its source loaded Q and by a 'geometry factor', the ratio of the 
effective shunt resistance $R_{\mathbf{r}}$ and its unloaded $Q$, i.e. $R / Q$.

The if component of beam image current, close to twice the dc beam current, is introduced on equal footing with the generator current, as shown.

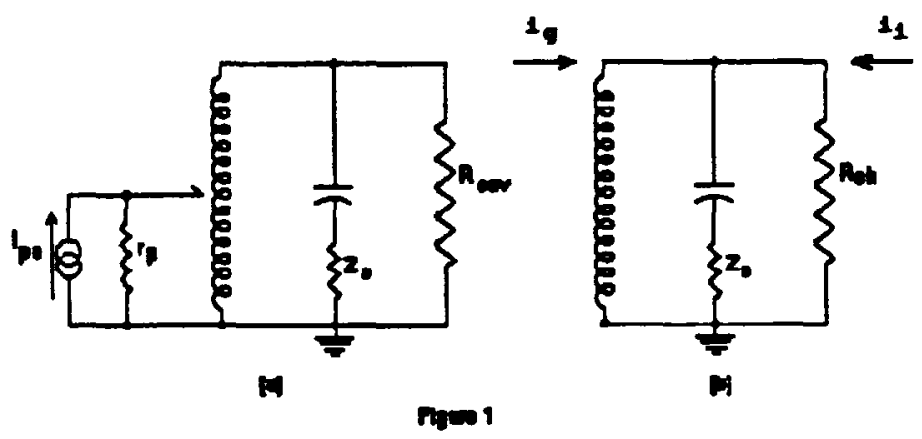

With the cavity tuned to resonance in the absence of beam current, a generator peak if current $i_{o}$ is defined to be the current necessary to create a voltage $V_{B}$ in phase with the generator current, i.e. $V_{2} \equiv i_{0} R_{n}$. This is shown in Fig. 2a where the phase of the beam $r$ current is chosen to be the positive real axis. (Below transition a stationary bucket is generated by gap voltage on the negative imaginary axis.) Using this convention the generator current $i_{0}$ and the gap voltage $V_{8}$ are shown at a synchronous phase angle $\phi_{\mathrm{r}}$. Fig. $2 \mathrm{~b}$ shows the same gap voltage being developed by a larger generator current in the presence of substantial beam and image current. The cavity has been tuned above its excitation frequency by an angle $\theta$ so that the voltages developed by the generator current and the image current lead each of the currents by the detuning angle $\theta$. By adjusting $\theta$ the load presented to the power amplifier can be made to appear real as shown.

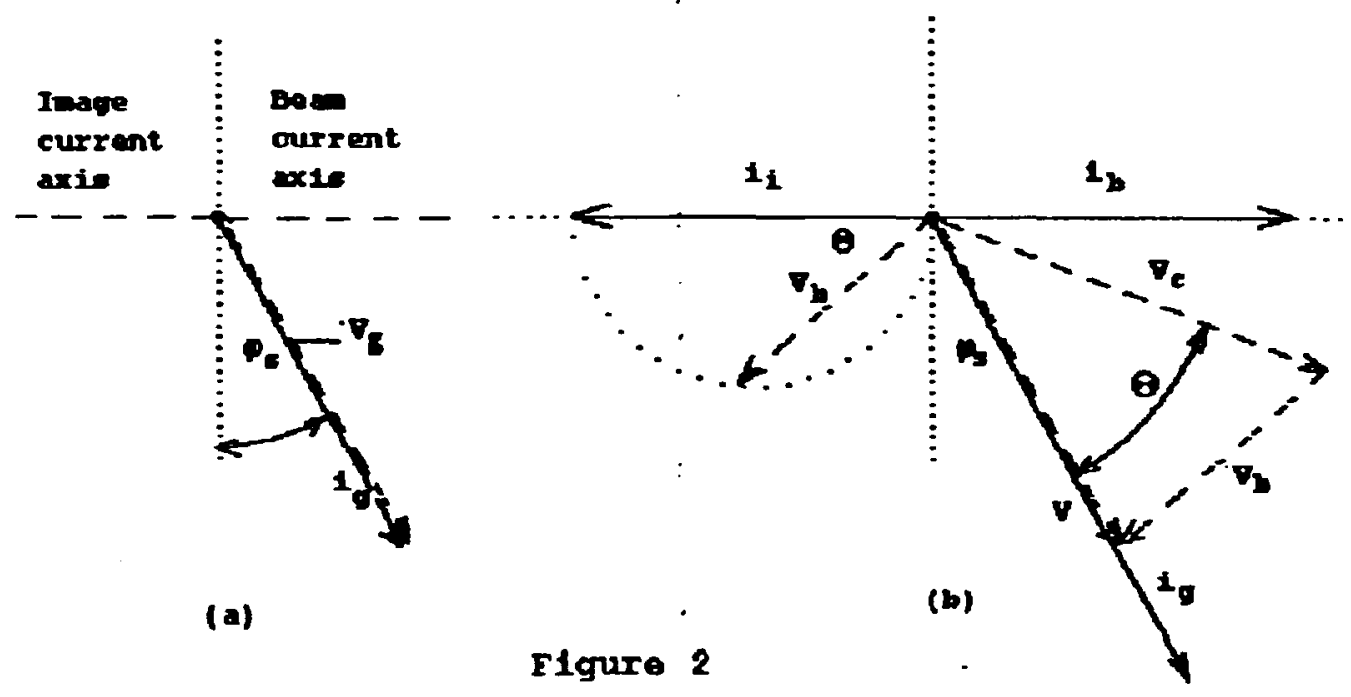


Near resonance the impedance of the cavity can be expressed:

$$
Z=R_{s h} \cos \theta e^{i \theta}, \quad \theta=\tan ^{-1}\left(2 Q \frac{\left(\omega_{0}-\omega\right)}{\omega_{0}}\right) .
$$

Here $\left(\omega_{0}-\omega\right)=\Delta \omega$ is defined such that $\theta$ represents the 'impedance' angle of the resonator. The detuning angle and generator current necessary to cause the amplifier load to appear real in the presence of beam current are:

$$
\begin{gathered}
\tan \theta=\left[\frac{\dot{b}}{\dot{j}}\right] \cos \phi_{s} \quad \operatorname{or} \quad \tan \theta=\left[\begin{array}{c}
i_{b} R_{s} \\
V_{g}
\end{array}\right] \cos \phi_{s} \\
\dot{g}=i_{o}+i_{b} \sin \phi_{s} .
\end{gathered}
$$

The increase in generator current is just that required for the generator to deliver the required power to the beam in addition to that dissipated in the cavity. These expressions are linear idealizations because the increase in generator current necessary to keep the gap voltage constant under beam loading will increase the dissipation in the power source so that the effective $\mathbf{R}_{\boldsymbol{w}}$ will be decreased; i.e. $\mathbf{R}_{\mathrm{th}}$ cannot be considered to remain constant. For this reason two forms of the equations are presented in (2) above.

\section{BEAM DRIVEN INSTABILITY, $M= \pm 1^{[4]}$}

The cavity frequency shift $\Delta \omega / \omega_{0}$ required to present a real load to the power source is of interest when compared to the frequency increment between the if frequency and the nearest rotation harmonics $(h \pm 1) \Omega_{0}$. Using the $\tan \theta$ expression from Eq. 2 and the cavity voltage $\left(V_{3}=i_{0} R_{2}\right)$ it is clear that the frequency shift is minimized by reducing $R / Q$ and operating each cavity at the maximum possible voltage:

$$
\begin{gathered}
\tan \theta=2 Q\left[\frac{\Delta \omega}{\omega_{0}}\right]=\left[\frac{i_{b} R_{s h}}{V_{g}}\right] \cos \phi_{s} \\
\frac{\Delta \omega}{\omega_{0}}=\frac{1}{2 V_{g}}\left[\frac{R}{Q}\right]_{i_{b}} \cos \phi_{s} .
\end{gathered}
$$

Although $R / Q$ remains constant the frequency shift is proportional to the beam current $i_{b}$ and to $\cos \phi_{f} / \mathrm{V}_{\mathrm{g}}$. Very large beam current may cause the frequency shift to become a large fraction of the rotation band spacing. In Fig. 3 resonance curves are shown for two values of $Q$ typical of those which might be obtained in a relatively low $Q$ ferrite tuned if cavity. The curves are plotted on a 
frequency scale which represents the position of the nearest rotation band frequencies (where the harmonic number is assumed to be substantially larger than 1).

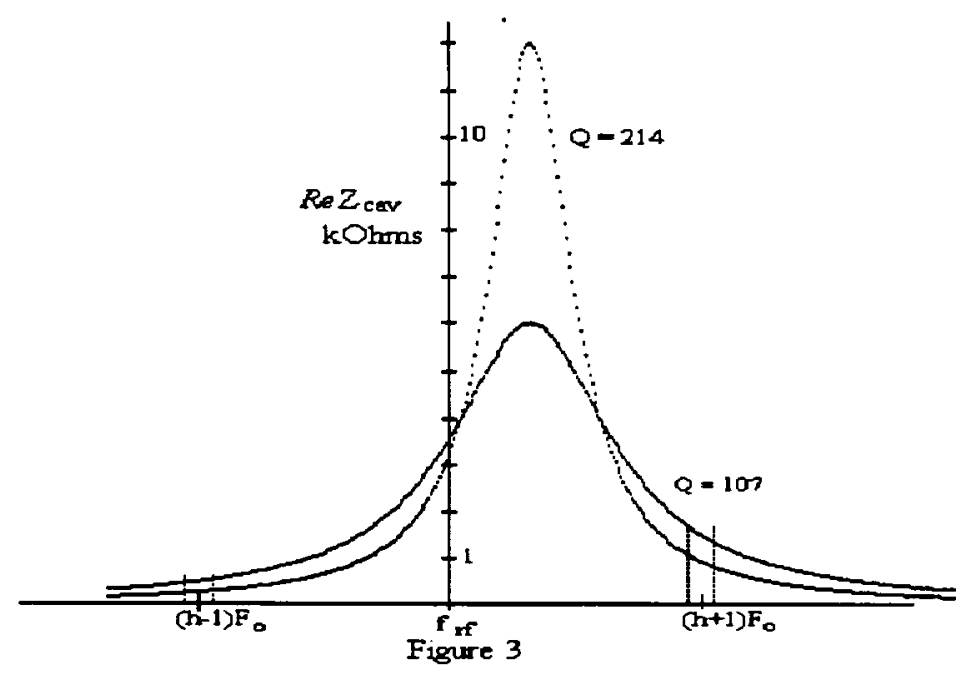

With the cavity excited below resonance, as required for real power amplifier loading below transition, the slope of the resonance curve is positive at the if frequency. This creates the condition that the real impedance seen by the beam at the upper synchrotron oscillation frequency is larger than that seen at the lower oscillation frequency. Therefore, in addition to providing the desired loading condition, this is the correct condition (below transition) for establishing Robinson stability (see below). However the stability condition is reversed at the $h \pm 1$ rotation frequencies where the impedance at the lower synchrotron sideband of $(h+1)$ is larger than at the upper sideband of $(h-1)$. This is the condition for growth of a mode 1 beam induced instability wherein each bunch executes coherent harmonic oscillation within its bucket with the relative phase of the oscillations progressing one complete cycle per turn. The opposite is true of the outer synchrotron sidebands of the rotation harmonic where the ratio would contribute a damping term to the mode -1 oscillation. But the ratios are such that the growth term dominates. In this way a particular combination of harmonic number, rotation band spacing, and cavity Q-related bandwidth can generate an unacceptably rapid growth of mode \pm 1 instability, which, in turn, contributes to the growth of longitudinal emittance during the acceleration cycle. 


\section{ERACTIONAL DETUNING}

While real load detuning is easily implemented and it minimizes anode dissipation for a given If load, it may be expedient to explore a scheme which results in smaller detuning. Cavity detuning can be reduced by adjusting the cavity frequency such that the generator current is not in phase with the anode voltage (i.e. the load on the tube is not 'real'). This amounts to introducing into the cavity drive current $i_{8}$ a component of current opposite to the beam image current $i_{i}$ which directly compensates for a fraction of the beam curent. In Fig. 4 the voltage and current phasors are shown with the generator current $i_{8}$ leading the cavity gap voltage $V_{g}$ by an angle $\Delta$.

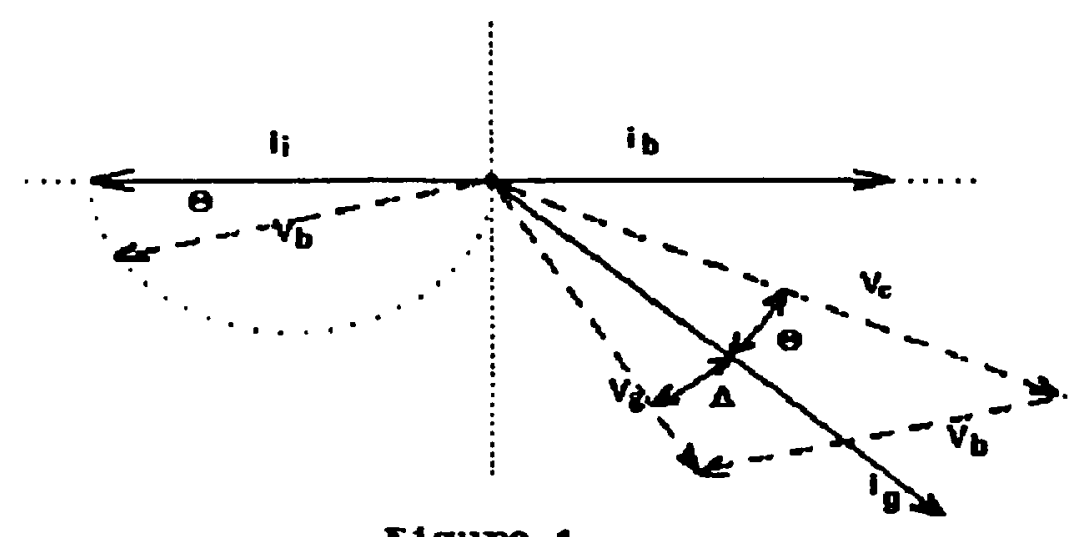

F1gure 4

Anode dissipation increases by a factor approaching 2.6 as $\theta$ approaches $\pi / 4$. (cf. Appendix 2). The effective cavity $Q$ and $R_{\text {th }}$ are decreased by the inverse of the same factor. For future use the reduction factor is labelled $\mathrm{D}$. (D $\leq 1)$. The detuning angle and the generator current now become:

$$
\begin{aligned}
\tan \theta & =\left[\frac{i_{b} D R_{s h}}{V_{g}}\right] \cos \phi_{s}-\tan \Delta\left(1+\left[\frac{i_{b} D R_{s h}}{V_{g}}\right] \sin \phi_{s}\right) \\
i_{g} & =\sec \Delta\left(\frac{V_{g}}{D R_{s h}}+i_{b} \sin \phi_{s}\right) .
\end{aligned}
$$

If $\Delta=0$ these expressions revert to Eqs.2.

Because the choice of $\Delta$ is somewhat arbitrary it is interesting to set it equal to $\theta$. The detuning parameters become: 


$$
\begin{gathered}
\tan \theta=\frac{i_{b} D R_{s h} \cos \phi_{s}}{2 V_{s}+i_{b} D R_{s h} \sin \phi_{s}} ; \quad i_{s}=\sec \theta\left(i_{0}+i_{b} \sin \phi_{i}\right) \\
\text { and } \Delta f=\frac{6}{2 D Q} \tan \theta .
\end{gathered}
$$

(Note: In these expressions $R_{d}$ represents the effective cavity shunt impedance which would obtain with the system detuned to present a 'real' load to the amplifier. The factor $\mathrm{D}$ applies the additional reduction caused by fractional detuning. The effective $R_{\text {m }}$ is obtained by calculating the anode dissipation and $r_{p}$, placing $r_{p}$ in parallel with the cavity driving point impedance, then transforming the result to the gap by a factor of four.)

Fractional detuning, to the extent that anode dissipation limitations will allow it, will reduce the frequency shift. Because $D$ is a function of $\theta$ some iteration is required to arrive at acceptable values of $\Theta$ and $\Delta f$.

\section{ROBINSON INSTABILITY}

In an early paper on beam-cavity stability ${ }^{[3]}$ Ken Robinson set out some stability conditions which are to be met in the absence of impedance controlling feedback. Even in systems which employ various feedback loops of differing bandwidth it is useful to examine these stability conditions. The stability limits are;

$$
0<\sin 2 \theta<2\left[\frac{i_{0}}{i_{b}}\right] \cos \phi_{5},
$$

where $\theta$ is the impedance angle of the $\mathrm{ff}$ cavity. The first inequality states that, below transition, the condition for stability is that the if cavity must be tuned above the excitation frequency, i.e. it must look like an inductance to the excitation currents. Above transition (Robinson's case for an electron accelerator) the opposite is true. If the if system is detuned such that the load appears real to the power generator, as described above, then the first of the stability requirements is automatically met. This is usually done by a feedback system which compares the phase of the generator current to that of the gap voltage and adjusts the cavity tuning for a (nearly) real load condition. Partial detuning of the proper sign will suffice.

If the cavity is detuned to create a real power source load (Eq.2), then the second inequality can be shown to state that the power dissipated in the rf system must be equal to or greater than that delivered to the beam. This condition is not actually a threshold for instability except for the unreal 
case where the longitudinal emittance of each bunch approaches zero, i.e. all of the charge is concentrated at a point within the bucket. This is because it can be shown that as the power ratio approaches unity the bucket area approaches zero. The reduced bucket area will at some point cause beam loss until the bucket is full and the condition for stability is met at reduced beam current. This rather simple concept is clouded and somewhat ameliorated if the system is 'fractionally detuned'.

By starting with fractional detuning and introducing a small perturbation of the beam phase angle, a 'reduction factor' $\mathrm{F}$ can be derived which can be applied to bucket area, bucket height, or phase oscillation frequency.

$$
F=\left[1-\frac{i_{b} D R_{s h} \sin \theta \cos \theta}{V_{g} \cos \phi_{s}}\right]^{2}
$$

This factor is equivalent to the second of Robinson's inequalities if the detuning factor $D$ is equal to one. The factor can be expressed in terms of accelerating voltage $V_{B}$, the beam current $i_{b}$, the phase angle $\phi_{v}$ and the reduction factor $D$. It is interesting that bucket factors are diminished even for a stationary bucket where $\phi_{\mathbf{r}}=0$ (or $\pi$ ) and no power is being delivered to the beam. For example if the beam current is equal to $i_{o}$ and $\phi_{0}=0$ bucket factors are reduced from their no-beam values by the factor 0.77 . From this point of view the Robinson inequality establishes (for the correct sign of detuning) a region of 'instability' with a fuzzy boundary. At a particular beam to cavity current ratio the system may be stable only for relatively small detuning. Large angles may then cause serious bucket reduction. Detuning angles larger than one radian tend to encroach seriously on the instability region ${ }^{[6]}$.

S. Koscielniak ${ }^{[\eta]}$ has expanded this notion to non-linear particle motion within the bucket and developed the notion of 'coherent' and 'incoherent' buckets. An 'incoherent' bucket is a phase space separatrix created primarily by the beam current in such a way that the beam remains bunched but the azimuthal location of the bunches is not locked to the if phase. Presumably the beam is being decelerated by the resulting if phasor and will very soon be lost against the vacuum chamber while still somewhat bunched.

\section{TRANSIENT BEAM LOADING}

At high intensity it will be necessary to maintain a gap of a few tens of nanoseconds in the beam to provide for extraction kicker risetime. With many bunches in the beam (high harmonic number) this indicates a series of two or three adjacent empty buckets. If the generator current is not 
reduced and/or shifted in phase (possibly by fast feedback loops) the excess cavity excitation will cause the gap voltage phase and amplitude to change such that the bunches immediately following the gap will be mismatched in phase and amplitude with respect to the buckets. This can constitute an additional source of emittance dilution. In order to evaluate the possible severity of this effect in the absence of effective feedback, we can calculate approximate changes in gap voltage amplitude and phase in two steps. During the period when the cavity is not being excited by the fundamental Fourier component of beam current we assume, first, that the stored energy in the cavity due to the gap voltage $\mathrm{V}_{\mathrm{g}}$ is decaying exponentially at the cavity resonant frequency. Secondly, we can calculate the voltage and phase that the existing generator current $i_{g}$ would generate in an empty cavity during the same period. The resultant voltage at the end of the gap will be the sum of these two phasors.

Because the cavity resonant frequency $\omega_{0}$ is not equal to the beam if frequency $\omega$, voltage phasors in the phase diagram containing the beam current must be expressed in terms of $\omega$. Below transition it is convenient to define $\Delta \omega=\left(\omega_{0}-\omega\right)$. The decaying cavity voltage $v(t)$ is:

$$
\begin{gathered}
v_{g}(t)=V_{g} e^{-\alpha t} e^{i \omega_{0} t}=V_{g} e^{-\alpha t} e^{i \omega t} e^{i\left(\omega_{o}-\omega\right) t} \\
=e^{-\alpha t} e^{i(\omega+\Delta \omega) t} \quad \text { where } \alpha=\frac{\omega_{o}}{2 D Q}
\end{gathered}
$$

This is a voltage phasor which starts at the original phase angle of $\mathrm{V}_{\mathrm{g}}$ (not included in the expression for clarity) and rotates clockwise with the time varying phase angle $\Delta \omega$ while decaying in amplitude with time constant $\alpha$.

To this must be added the phasor which results from excitation of the cavity by the generator current $\mathrm{i}_{\mathrm{g}}$. This can be obtained by first obtaining the response of the resonant circuit to delta function, then calculating the convolution of a phasor expression for the generator current with the delta function response (Green's function). The result, again expressed in terms of the beam frequency $\omega$ and dropping higher order terms, becomes:

$$
\begin{aligned}
& v_{g}(t)=I_{g} R_{s h} \cos \Theta e^{i \theta} e^{i \omega t}\left[1-e^{-(\alpha-\Delta \omega) t}\right] \\
& \approx \frac{I R_{s h} e^{\omega \omega t}}{1-i \frac{\Delta \omega}{\alpha}}[\alpha-i \Delta \omega] t=I_{g} e^{j \omega t}\left(\frac{\omega_{0} R_{s}}{2 Q}\right) t .
\end{aligned}
$$


For small $t$ this is a phasor growing linearly with time in the direction of the current $i_{r}$. Under heavy beam loading conditions and with the possibility of enhanced generator current resulting from fractional detuning, this phasor will be substantially larger than the decrement resulting from decay of the starting gap voltage. Assuming fractional detuning, where the generator current leads the gap voltage (below transition) by $\theta$, the cavity voltage at the end of a beam gap of time $t$ will be:

$$
V_{c o v}=V_{g} e^{-\alpha t_{e}} e^{\left\langle\phi_{1}-\frac{\pi}{2}+\omega_{0} \theta\right.}+\left(\frac{I_{g} \omega_{0} R_{s}}{2 Q}\right) e^{i\left(\phi_{j}-\frac{\pi}{2}+\theta\right)} t
$$

Since it appears to be possible to keep the detuning angle rather small, this effect may appear primarily as a change in bucket height during the beam gap.

\section{EEEDBACK SYSTEMS}

The stability limits described above refer to 'open loop' rf accelerating systems in which there is no attempt to stabilize the accelerating voltage amplitude and/or phase by feeding a sample of the gap voltage back to some point in the power drive chain to correct for beam driven deviations by changing the drive current amplitude or phase. Such a system could, in effect, reduce the output impedance of the $f$ cavity system in standard feedback manner.

Essentially all modern $\mathrm{rf}$ accelerating systems have some feedback systems installed for various reasons ${ }^{[8,9,10]}$. Such systems may or may not be effective in reducing beam driven deviations depending on loop gain, bandwidth and configuration. For example, a system which compares the drive current phase to the gap voltage phase for the purpose of detuning the cavity in order to cause the average load to appear 'real' will almost certainly have insufficient bandwidth to compensate for deviations at the phase oscillation frequency.

While a properly configured feedback system may contribute beam stability under heavy beam loading by reducing the effective output impedance of the accelerating system, the power and current delivery requirements of the system will not be reduced significantly. In order to accomplish the impedance reduction, the system must be capable of delivering generator current $i_{g}$ at least equal to expected transient deviations in beam current to the accelerating gap. Otherwise a large error signal from the feedback summing junction may saturate the power amplifier at some point. Therefore, while certain of the instability limits described above may be relaxed or ameliorated through the use of feedback, it is nevertheless useful to examine the power and current delivery capability of an if system in the light of expected beam current and power. 


\section{LONGITUDINAL SPACE CHARGE EFFECTS}

The charge distribution in the machine during acceleration can be characterized at some instant in time by a function of distance along the orbit, $\lambda(z)$. The Fourier components of the image charges of $\lambda(z)$ moving with speed $\beta c$ will interact with the beam pipe and/or other components in the vacuum chamber creating fields which can deposit energy in the chamber and otherwise affect the accelerating gradient or bucket parameters. In general the fields created by Fourier components of the beam which react back on the beam can be characterized by the product of these components and a frequency dependant impedance $Z(\omega)$. The fields and equivalent ring voltages generated in the reactive part of the vacuum chamber impedance are proportional to the derivative of the line charge distribution ${ }^{[11]}$.

$$
E_{z}=e \beta^{2} c^{2} \frac{\partial \lambda(z)}{\partial z} \frac{d L}{d z} \quad \text { and } \quad V_{z}=-e \frac{\partial \lambda(z)}{\partial z}\left[\frac{R_{s} g_{o}}{2 \epsilon_{o} \gamma^{2}}-L \beta^{2} c^{2}\right] \text {, }
$$

where $R_{s}$ is the mean orbit radius, $\epsilon_{o}$ is the permittivity of free space, and $L$ is the effective inductance of the vacuum chamber. For circular beam pipe $g_{o}=(1+2 \ln (b / a))$, where $b$ is the chamber radius and $a$ is the mean beam radius. Parabolic line charge distributions are commonly used for proton bunches but it is also reasonable to use a $\cos ^{2}$ distribution (parabolic at its center but with longer tails). If $\mathrm{N}$ is the number of particles per bunch and $\mathrm{l}$ is the full bunch length the $\cos ^{2}$ distribution can be written:

$$
\lambda(z)=\frac{2 N}{l} \cos ^{2}\left(\frac{\pi z}{l}\right) ; \quad \frac{\partial \lambda}{\partial z}=-\frac{4 \pi N}{l^{2}} \sin \left(\frac{2 \pi z}{l}\right), \quad-l / 2 \leq z \leq l / 2
$$

Fields resulting from the inductive wall part of Eq. 11 add to the effective bucket height and cause bunch shortening below transition. Above transition the opposite is true. 


\section{FERMILAB BOOSTER}

The Fermilab $8 \mathrm{GeV}$ Booster has mean orbit circumference $474.2 \mathrm{~m}, \gamma_{\mathrm{t}}=5.446$, and it operates at harmonic number 84 . The ring contains 96 combined function magnets.

The Booster has no vacuum chamber within the magnets. The beam image charges impinge directly on magnet laminations, a situation which drastically increases the ring impedance presented to the beam. Calculations by S. Snowden and A. Ruggiero ${ }^{[12,13]}$, and measurements by F. Mills ${ }^{[14]}$ and $\mathrm{J}$. Crisp ${ }^{[15]}$, indicate impedances ranging from 10 to $100 \mathrm{k} \Omega$ in frequency range 25 to $100 \mathrm{MHz}$ with about equal real and reactive parts. There are large uncertainties and variations in both the calculations and the data. This situation constitutes a serious limitation on operation of the Booster at very high intensity unless some sort of conducting strips or wires can be inserted into the vacuum chamber of all magnets.

A single magnet has been fitted with conducting strips along the magnet pole faces. Impedance measurements indicate a total resistive component of about $2 \mathrm{k} \Omega$ up to $200 \mathrm{MHz}$, but the measured reactive component was $8 \mathrm{k} \Omega$ and apparently capacitive at all frequencies. This measurement was probably in error and should be explored further.

The Booster if system has 16 (expandable to 18) ferrite tuned if cavities capable of operation between 30 and $53 \mathrm{MHz}$. The if cavities are double gap structures with gap spacing 140 degrees (or $0.389 \lambda$ ). The total accelerating voltage generated by a cavity is $V_{g}=2 V_{1 g} \sin (70)=1.88 V_{1 g}$, where $V_{1 g}$ is the voltage developed at each gap. The rf voltage step-up from the tube anode to one gap is approx. 1.1, so we assume here the step-up from anode to effective accelerating gap to be 2 . The Booster if cavities have been upgraded (called 'super cavities') ${ }^{[16]}$ and indications are that each cavity is capable of developing $30 \mathrm{kV}$ at each gap $\left(\mathrm{V}_{\mathrm{g}}=56 \mathrm{kV}\right)$ at frequencies greater than $40 \mathrm{MHz}$.

Each cavity is powered by a single Eimac 4CW150,000 (Y567B) water cooled tetrode rated for continuous anode dissipation $150 \mathrm{~kW}$. The cathodes of the tubes are capable of delivering peak current of $150 \mathrm{~A}$. At present the ' $160 \mathrm{~kW}$ ' power tetrodes are anode modulated with the 'old' series tube modulators, capable of programming the anode voltage up to around $25 \mathrm{kV}$ and delivering up to $12 \mathrm{~A}$ average dc current. The tubes are cathode driven with the control grid grounded. Data relating the average (dc) cathode current to the peak value of the fundamental Fourier component of if current delivered to a real load indicate that this ratio is about $1: 1.56$. These data were obtained by measurement of the if power spectrum developed when the tube current was delivered to a water cooled $50 \mathrm{Ohm}$ load ${ }^{[17]}$. Analysis of the cathode current wave in a more realistic circumstance (cf. Appendix B) where the anode voltage is allowed to swing down to a few $\mathrm{kV}$ indicates that this ratio 
is more like 1:7. Using these data the anode dissipation can be calculated directly for any average cathode current. If the tube anode resistance $r_{p}$ is defined by $r_{p}=V_{r f}^{2} /(2 P$ mode $)$ then $r_{p}$ ranges from near $1 \mathrm{kOhm}$ to $23 \mathrm{kOhms}$ depending on the power being developed by the tube. In most operating conditions where the cavity is detuned to present a real load to the amplifier the anode dissipation is smaller by a factor of about two than the power delivered to the load. This implies that the value of $r_{p}$ to be considered in parallel with the loaded driving point impedance is larger by the same factor than the load impedance. If the system is 'fractionally detuned' the anode dissipation is increased substantially and the effective $r_{p}$ lowered accordingly (cf. Appendix B).

The $Q$ of the cavity is dominated by the $Q$ of the ferrite used to tune the cavity. In the frequency range of interest the ferrite is quite heavily biased, raising the $Q$ to near its maximum value. We assume for this note that the $Q$, not loaded by the power amplifier anode resistance, is 500. Because of the vicissitudes of ferrite as a function of frequency and field amplitude, it is difficult to ascribe to a ferrite loaded cavity (especially a double gap cavity) a 'geometry factor' $R / Q$. However it may be useful to define such a factor for this cavity in terms of its unloaded properties. At frequencies near $45 \mathrm{MHz}$ one could assign this system $\mathrm{R} / \mathrm{Q}$ to be approximately 56 .

The driving point impedance of the cavity (that impedance presented to the power amplifier in the absence of beam loading) varies from a few kOhms at $30 \mathrm{MHz}$ to $8 \mathrm{kOhms}$ at $53 \mathrm{MHz}$. For the frequency range of interest here we assume $7 \mathrm{kOhms}$. This impedance, transformed to the accelerating gap, results in an 'unloaded' shunt impedance of $28 \mathrm{kOhms}$. For any effective peak gap If voltage this value can be used to approximate the (primarily ferrite) cavity power dissipation. $P_{c o v}=V_{8}{ }^{2} / 2 R_{d}=V_{8}{ }^{2} /\left(56 \times 10^{3}\right) \mathrm{W}$. The $7 \mathrm{kOhm}$ driving point impedance appears in parallel with the effective tube anode resistance $r_{p}$ when cavity $Q$ or time constant is required under driven conditions. The tube anode dissipation should be calculated separately using the expression from Appendix B.

The entire complex of if stations receives an if drive signal of the correct frequency and phase from a 'global' low-level feedback system which uses the beam phase as its information source.

Each if station has two 'local' feedback loops. The first loop compares the amplitude of the if gap voltage to a pre-programmed level and adjusts the $\mathrm{dc}$ current through the tube to maintain the correct level. This is done by detecting a rise in the tube screen grid current when the anode if voltage swings near the dc screen voltage $(\sim 1500 \mathrm{~V})$. The if drive level remains constant. The maximum if drive capability ( 1.7 times the average dc current) is around $18 \mathrm{~A}$ peak $\mathrm{rf}$ current. This loop is relatively fast and can probably compensate for amplitude changes caused by the beam at the synchrotron frequency. 
The second 'local' feedback loop was originally intended to compare the anode (or gap) if voltage phase with the cathode drive current phase, using the error signal to adjust the cavity ferrite bias current such that the cavity is detuned to establish a 'real' load. Because the total inductance of the ferrite is quite large the bandwidth of this loop is only a few Hertz.

This feedback loop has been subverted in the Booster such that the gap voltage is compared not to the drive current phase but rather to the phase of the low level signal supplied to the station ahead of the low power driver amplifiers. In this way the cavities are detuned such that the cavity gap voltage phase is correct and phase errors in the driver amplifiers are compensated for. The cavities are effectively being used as high power phase shifters. This is not a good situation because the potential exists for the balance of the cavities to be detuned in such a way as to be Robinson unstable early in the cycle during adiabatic capture following injection. The situation could be turned to advantage however, by inserting in each drive line a low power voltage controlled phase shifter which is driven by the error signal. This would introduce into each station a relatively broad-band phase feedback system in addition to the existing amplitude feedback. Also the cavity detuning system could be reinstalled as originally intended.

New series tube modulators with substantially enhanced current capability are being installed in the Fermilab Main Injector. At the same time the vacuum tube cathode driver amplifiers are being replaced by solid state amplifiers. These two changes will improve the power delivery capability of the if systems. It is reasonable to assume that when that project is finished some version of the improvements can be applied to upgrading the Booster if systems. For extremely high intensity operation the power and voltage capability of new solid state driver amplifiers may have to be somewhat larger than those presently being installed, but such a design change is not unreasonably difficult.

\section{HIGH CURRENT OPERATION}

For injection energy l GeV ( $\beta=0.875)$ the if frequency range is 46.47 to $52.813 \mathrm{MHz}$. The rotation period at injection is $1.81 \mu \mathrm{s}$. With $5 \times 10^{13}$ protons in the ring the average current at injection will be $4.42 \mathrm{~A}$. For reasonably tightly bunched beam the if beam current will be near $8 \mathrm{~A}$ At extraction the $\mathrm{rf}$ component of beam current will approach $10 \mathrm{~A}$. We assume total injected longitudinal emittance to be $2 \mathrm{eV}-\mathrm{s}$. At the end of adiabatic capture we expect the longitudinal emittance of each bunch to be near $0.03 \mathrm{eV}$-s.

Fig. 5 is a set of curves showing the required accelerating voltage $V_{\infty}=V_{8} \sin \varphi_{1}$, the total voltage necessary to provide $0.12 \mathrm{eV}$-s bucket area during the acceleration cycle, and the resultant 
synchronous phase angle. The reduction factor $\mathrm{F}$ is not included. The maximum voltage requirement, $760 \mathrm{kV}$, occurs near $6 \mathrm{~ms}$. If updated modulators are in place each cavity could produce $56 \mathrm{kV}$ and only fourteen cavities would be required. However, beam power considerations later in the cycle indicate otherwise.

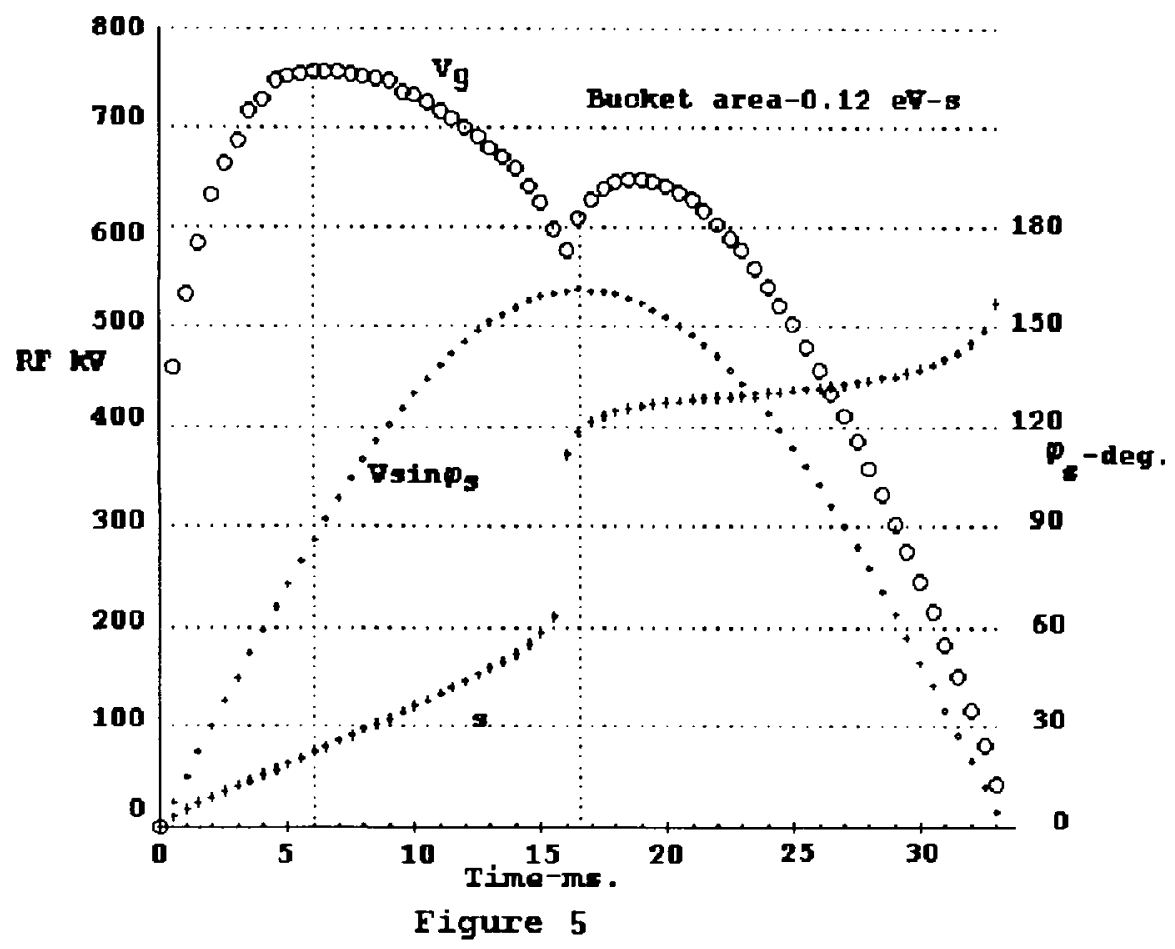

Detuning at $6 \mathrm{~ms}$, mode \pm 1 coupled bunch problem

With eighteen cavities operating, $42 \mathrm{kV}$ per cavity will provide the $760 \mathrm{kV}$ required at $6 \mathrm{~ms}$. The synchronous phase angle is $22.2 \mathrm{deg}(0.39$ radians $)$ and $V_{\text {acc }}$ is $287.2 \mathrm{kV}$. The rf frequency is $51.15 \mathrm{MHz}$ and the peak beam $\mathrm{rf}$ current is $9.75 \mathrm{~A}$. The power delivered to the beam is $1.4 \mathrm{MW}$ ( $77.8 \mathrm{~kW}$ per cavity). Each cavity will dissipate $31.8 \mathrm{~kW}$ so the total if power requirement per cavity will be $110 \mathrm{~kW}$. Under real load tuning the anode dissipation is $36 \mathrm{~kW}, \mathrm{r}_{\mathrm{p}}$ is $6.2 \mathrm{kOhms}$, and the effective $\mathrm{R}_{\mathrm{sh}}$ is $13 \mathrm{kOhms}$. The total $\mathrm{ff}$ source dissipation will be $67.8 \mathrm{~kW}$ so the ratio of beam to source power becomes 1.15 , which may be a bit high depending on the efficacy of feedback systems. None of these parameters appear to present any problem for a Booster if station other than possible stability problems

From Eq.2, $\tan \Theta=2.8, \Theta=70.4 \mathrm{deg}$. (1.23 radians), which is excessive and almost certainly unstable. The resonant frequency shift, Eq. 3 , is $308 \mathrm{kHz}$, slightly more than half of the frequency increment to the adjacent $(\mathrm{h}+1)$ rotation band, $609 \mathrm{kHz}$. This presents the possibility of unacceptable mode +1 instability growth rate. 
If the system is operated with fractional detuning, Eq.(6), the effective $Q$ and $R_{\text {sh }}$ are reduced to 137 and $7.7 \mathrm{kOhms}$ respectively. (The reduction factor $\mathrm{D}$ is 0.589 .) The anode dissipation is increased to $61 \mathrm{~kW}$ and the ratio of source power to beam power is increased to 1.2. The detuning angle $\Theta$ is $31.6 \mathrm{deg}$. ( 0.55 radians) and the frequency shift is reduced to $115 \mathrm{kHz}$. The Robinson bucket reduction factor, Eq.(8), is 0.38 whereas without fractional detuning it was zero. All of these changes are within the capability of a Booster if station and they are favorable with regard to beam stability. In Fig. 6 the cavity resonance curves are shown for the two tuning conditions. The lower $Q$ curve has moved closer to the rf frequency and the ratio of its real component at the nearest rotation harmonics is decreased even though the curve is broader than the higher $\mathrm{Q}$ curve.

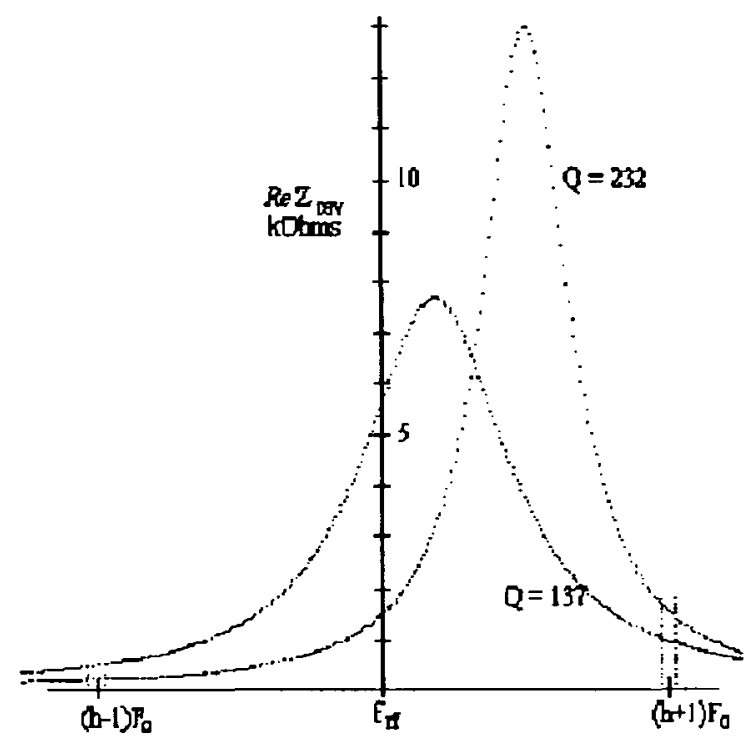

Figure 6

Maximum beam power point, $16.6 \mathrm{~ms}$.

The maximum accelerating voltage, $\mathrm{V}_{\mathrm{g}} \sin \varphi_{\mathrm{s}}$, is $536 \mathrm{kV}$ at $16.67 \mathrm{~ms}$. With $5 \mathrm{~A}$ dc beam current the power delivered to the beam at this time is $2.68 \mathrm{MW}, 149 \mathrm{~kW}$ from each of eighteen cavities. At $16.6 \mathrm{~ms}$ the total if voltage is $608 \mathrm{kV}$ so each of eighteen cavities must supply $33.8 \mathrm{kV}$. The additional $20.4 \mathrm{~kW}$ dissipated in each cavity will bring the total power requirement per cavity to about $170 \mathrm{~kW}$. From the curves it is evident that very little additional voltage is required at this time for bucket generation because $\gamma$ is very near $\gamma_{\mathrm{t}}$. The synchronous phase angle is near $118 \mathrm{deg}$.

With real load detuning the anode dissipation will be near $59 \mathrm{~kW}$ and the average anode 
current will be $11.8 \mathrm{~A}$ with dc anode voltage $19.4 \mathrm{kV}$. The detuning angle is $-44.6 \mathrm{deg}$. and the frequency shift is $-188 \mathrm{kHz}$. The total source dissipation, $\sim 80 \mathrm{~kW}$, is not sufficient by a large margin to exceed the power delivered to the beam so, in the absence of effective feedback, the bucket area will be reduced to zero. Because of the proximity of transition the bucket reduction factor may not be a matter of great concern here; the existing or proposed feedback systems may be adequate to establish stability in this situation.

With fractional detuning the detuning angle becomes $-13.5 \mathrm{deg}$. (0.237 radians) and the frequency shift is reduced to $-50 \mathrm{kHz}$. The bucket reduction factor F increases to 0.3 , indicating the need for at least some feedback to maintain adequate bucket area. The average anode current is increased to $12.2 \mathrm{~A}$, which is near the limit of presently used series tube modulators and vacuum tube cathode drive amplifiers.

\section{Transient Beam Loading Effects}

At the maximum beam power point in the cycle the generator current $\mathrm{i}_{\mathrm{g}}$ is $\sim 10 \mathrm{~A}$. The if voltage is $608 \mathrm{kV}$ at synchronous phase angle $\varphi_{\mathrm{s}} \sim 118$ degrees. With respect to the beam current phasor on the positive real axis the ring voltage can be expressed: $V_{g}=608 \mathrm{e}^{\mathrm{i} 0.489}$, or $608\left\lfloor 28^{\circ} \mathrm{kV}\right.$. Assume a gap of three unoccupied buckets, $56.6 \mathrm{~ns}$, is placed in the beam to accommodate extraction. If the generator current is unchanged during such a gap the ring voltage will change in amplitude and phase. Using Eq.(10), the voltage at the end of the gap will be $V_{g}=661\left[26^{\circ} \mathrm{kV}\right.$, i.e. the ring voltage will have increased by $53 \mathrm{kV}$ and moved in phase by 2 degrees. This would introduce a minor change $(\sim 5 \%)$ in bucket area and phase with a similar increase in longitudinal emittance of a few bunches following the gap. If the longitudinal emittance dilution resulting from such an effect is deemed to be unacceptable, the generator current could be adjusted by feeding a properly timed correction signal to the cathode drive amplifiers. The effect cannot be corrected by the existing amplitude feedback system because the round trip propagation time from the gallery to the cavities is greater than $100 \mathrm{~ns}$.

\section{Vacuum Chamber (or lack thereof) Effects.}

We examine the possible effects bunch excitation of the imaginary part of the Booster vacuum chamber impedance above transition. Measurements by J. Crisp ${ }^{[16]}$ indicate that the inductive part ot the ring impedance due to magnet laminations alone varies from 12 to $27 \mathrm{kOhms}$ between 50 and $75 \mathrm{MHz}$. In this range the beam current Fourier components are largest. Here we assume an average inductance per unit length $1.3 \times 10^{-7} \mathrm{Hy}$ per $\mathrm{m}$. along the orbit. The question is whether a confining separatrix (bucket) can be created above transition with this ring impedance. 
To generate the largest possible bucket above transition, set $\mathrm{ff}_{\mathrm{rf}}=800 \mathrm{kV}$ near $24 \mathrm{~ms}$. The accelerating voltage at that point is $400 \mathrm{kV}$ so $\varphi_{3}=150^{\circ}, \Gamma=0.5, \alpha(\Gamma)=0.333$, and the bucket length $\Delta \varphi=189^{\circ}$. The effective $E$ field seen by a particle in the coordinate system moving with a bunch is $\quad E_{z}=\left(\frac{V_{r f}}{2 \pi R_{s}}\right) \sin (\phi)=1.69 \times 10^{3} \sin \left(\frac{2 \pi z}{\lambda}\right)$ Volts per meter. ( $\mathrm{z}$ is the particle position.) The slope of the effective $E$ field, evaluated at $\varphi_{s}$ is:

$$
\frac{\partial E_{z}}{\partial z}=\frac{V_{g}}{R_{s} \lambda} \cos \phi_{s}=-1.64 \times 10^{3} \text { volts } / \mathrm{m}^{2} .
$$

This is to be compared with the voltage induced in the inductive reactance of the chamber by the inage charge of each bunch. We assume that each bunch $\left(\mathrm{N}=6.2 \times 10^{11}\right.$ protons) has a $\cos ^{2}$ line charge distribution (parabolic near its center) which spans $\lambda / 2$ at its base ( $\lambda$ is the rf wavelength, $5.6 \mathrm{~m}$ ). This nearly fills the putative bucket. The line charge and its derivative are:

$$
\lambda(z)=\frac{4 N}{\lambda} \cos ^{2}\left(\frac{2 \pi z}{\lambda}\right) \quad-\lambda / 4 \leq z \leq \lambda / 4 ; \quad \frac{\partial \lambda(z)}{\partial z}=-\frac{8 \pi N}{\lambda^{2}} \sin \left(\frac{4 \pi z}{\lambda}\right) .
$$

The field generated by this distribution moving through the chamber inductance is:

$$
E_{z}=e \beta^{2} c^{2} \frac{\partial \lambda(z)}{\partial z} \frac{d L}{d z}=-\frac{8 \pi e \beta^{2} c^{2} N}{\lambda^{2}}(L / m) \sin \left(\frac{4 \pi z}{\lambda}\right)
$$

The derivative of the beam induced field, evaluated at the center of the bunch is:

$$
\frac{\partial E_{z}}{\partial z}=\frac{32 \pi^{2} e \beta^{2} c^{2} N}{\lambda^{3}}(L / m) \cos \left(\frac{4 \pi z}{\lambda}\right)=2 \times 10^{3} \text { volts } / m^{2} .
$$

The positive slope induced by the bunch at its center is comparable to, but slightly larger than, the negative longitudinal restoring force necessary to create a bucket with the if voltage set at maximum. Therefore it is apparent that the booster cannot operate above transition at the proposed beam intensity without substantial modifications to the vacuum chamber. 


\section{CONCLUSIONS}

It appears evident from the above analysis that the Booster could not sustain beam intensity near $5 \times 10^{13}$ protons per cycle even if the measurements of vacuum chamber impedance are not precisely correct. Some sort of vacuum chamber or field shielding within the magnets will be necessary.

However, disregarding problems resulting from the high impedance vacuum chamber there appears to be no major obstacle to operation of the Fermilab Booster if system at beam intensity $5 \times 10^{13}$ protons per pulse with $2 \mathrm{eV}$-s injection at $1 \mathrm{GeV}$. The existing $150 \mathrm{~kW}$ power amplifier tubes are adequate to the task and no excessive anode dissipation or $\mathrm{ff}$ power generation are required. If the local feedback systems are not capable of maintaining stability the fractional detuning technique described can be introduced to reduce cavity $\mathbf{Q}, \mathbf{R}_{\text {th }}$, and frequency shift. These techniques can be employed without exceeding the rated dissipation. Some improvements over the existing system are indicated however.

1. The dc current capability of the existing series tube power modulators is not quite sufficient. The updated modulators now being installed in the Main Injector would be adequate.

2. The cavity detuning feedback system should be returned to its original configuration wherein the anode if voltage phase is compared to the cavity drive current phase and the cavity detuned accordingly. The error signal from the feedback system as it is presently configured may then be delivered to a voltage controlled phase shifter in the low-level fan-out line in order to correct and stabilize the phase of the accelerating if cavity voltage. This new feedback loop should have adequate bandwidth so that beam driven variations of the cavity voltage phase at the phase oscillation frequency can be minimized. In addition, some programmed phase shift wizardry will have to be invented to introduce the fractional detuning describe above.

3. A simple feed-forward system could be implemented to correct for excursions of the accelerating voltage during the necessary empty bucket gaps. This probably cannot be accomplished using feedback because of cable propagation times. 


\section{REFERENCES}

1. L.J. Laslett, Proc. International Symposium on Electron-Positron Storage Rings, Saclay, 1966 (Presses Universitaires de France, 1967) p.IV.5.

2. A.M Sessler, "Beam-Surrounding Interactions and the Stability of Relativistic Particle Beams", IEEE Trans. Nucl. Sci., NS-18, June 1971 (1039).

3. J. E.Griffin, AIP Conf. Proc. No.87, Physics of High Energy Particle Accelerators, 564, (1982).

4. A. Hofmann, "Single-Beam Phenomena-Longitudinal, Theoretical Aspects of the Behavior of Beams in Accelerators and Storage Rings", CERN 77-13, 139, (1977).

5. K.W. Robinson, "Stability of Beam in Radiofrequency System", Cambridge Electron Accelerator Report CEAL-1010, (1964).

6. F. Pedersen, "A Novel Cavity Tuning Feedback Sceme for Heavy Beam Loading", IEEE Trans. Nucl. Sci. NS-32. No.5, 2138, (1885.

7. S.R. Koscielniak, "Coherent and Incoherent Bucket for a Beam Loaded RF System", Jour. Part. Accel. 48, no.1 135-168 (1994).

8. F. Pedersen, "Beam Loading Effects in the CERN PS Booster", IEEE Trans. Nucl. Sci.,NS-22 1906, (1975).

9. F. Pedersen, "Beam Loading Aspects of the TRUUMF KAON Factory RF System", Triumf Report TRI-DN-85-15, (1985)

10. G.H. Rees, "RF System Design for Control of Heavy Beam Loading in Circular Machines" New Techniques for Future Accelerators 2, RF and Microwave Systems, Ettore Majorana International Science Series, M. Puglisi er al, Plenum Press 245-266, Erice (1987).

11. S. Hansen et.al., "Effects of Space Charge and Reactive Wall Impedance on Bunched Beams", IEEE Proc. Nucl. Sci., NS-22, no.3, 1381,(1975).

12. S.C. Snowden, "Wave Propagation Between Booster Laminations Induced by Longitudinal Motion of Beam", Fermilab TM-277, (1970)

13. A.G. Ruggiero, "Longitudinal Space Charge Forces Within Bunched Beams in the Presence of Magnet Laminations", Fermilab FN-220 and FN-230, (1971).

14. F. Mills, Private Communication, Measurement of shift in synchronous phase angle with beam current implies high real component of ring impedance.

15. J.L. Crisp, Private Communication, Wire measurements of Booster magnet impedances, implies frequency dependant impedances varying around $30 \mathrm{kOhms}$. Cf. J. Crisp for data.

16. Q.A. Kerns et.al., "30-53 MHz Cavities for $10 \mathrm{GeV}$ Acceleration in the Fermilab Booster Ring", IEEE Trans. Nucl. Sci. NS-26, no.3, 4111, (1979).

17. H.W. Miller, Private Communication, Fermilab, ca. 1980. 


\section{APPENDIX A: BOOSTER RING WITH GAMMA-T $=11$}

It is of interest to look at a $15 \mathrm{~Hz}$ ring with roughly the geometry of the Fermilab Booster but with $\gamma_{1}=11$. We assume that the ring operates at $h=84$ and has essentially the same $f$ capability as the existing Booster. The if voltage required to create individual bunch bucket areas $0.11 \mathrm{eV}-\mathrm{s}$ is plotted here against cycle time. Also shown are the accelerating voltage $V \sin \varphi$, and the synchronous phase angle. It is assumed that a vacuum chamber capable of reducing $Z / n$ to less than $50 \mathrm{hms}$ is in place and that the sysem is fractionlly detuned.

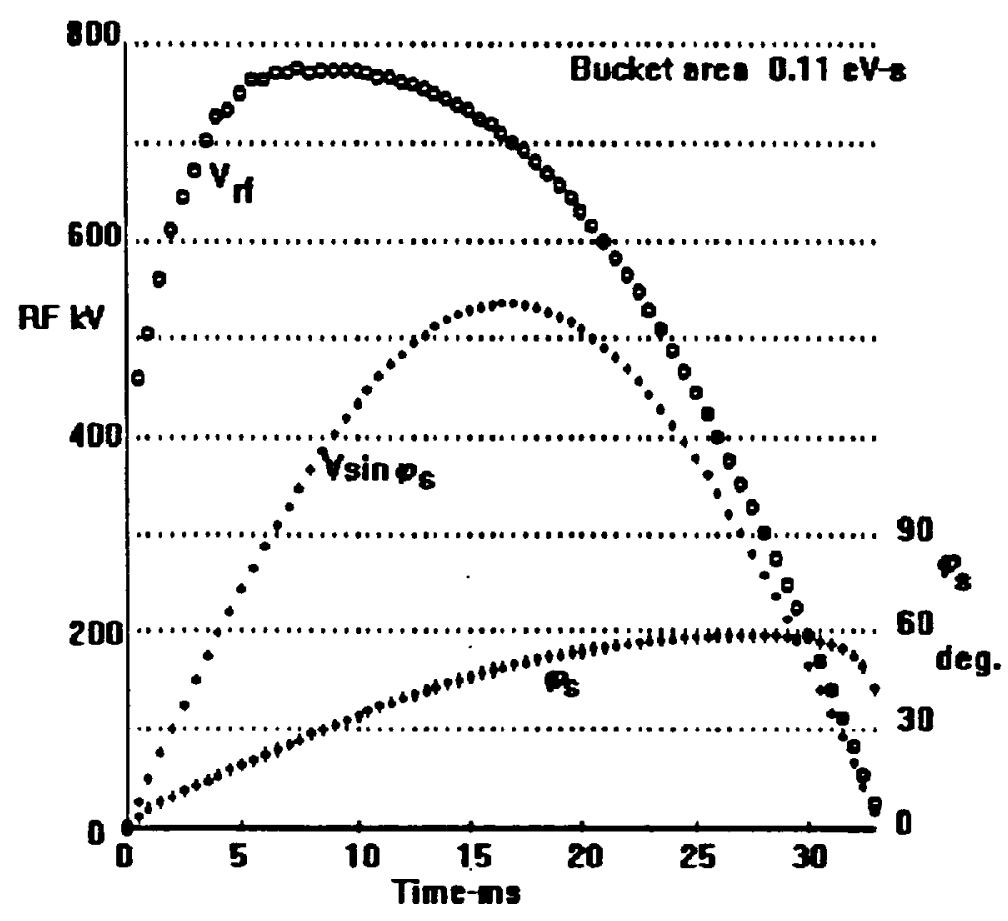

Two points in the cycle are of interest;

Maximum rf voltage is required at about $8.5 \mathrm{~ms}$.

$V_{\mathrm{rf}}=775 \mathrm{kV}$, or $43 \mathrm{kV}$ per cavity. (Presently available.)

$P_{\text {beam }} / \mathrm{cav}=95 \mathrm{~kW}, P_{\text {cav }}=33 \mathrm{~kW}$, Anode dissipation $=61 \mathrm{~kW}$

Detuning freq. shift $140 \mathrm{kHz}$.

Maximum power to beam is required at $16.67 \mathrm{~ms}$.

$\mathrm{V}_{\mathrm{rf}}=700 \mathrm{kV}$, or $39 \mathrm{kV}$ per cavity.

$P_{\text {beem }} /$ cav $=149 \mathrm{~kW}, P_{\text {ocv }}=27 \mathrm{~kW}$, (Total if power $176 \mathrm{~kW}$.)

Anode dissipation $71 \mathrm{~kW}$

Detuning freq. shift $71.3 \mathrm{kHz}$. 


\section{APPENDIX B - POWER TETRODE OPERATION WITH A REACTIVE LOAD}

In conditions of heavy beam loading it has been common practice to 'detune' the if cavity in such a way that the load presented to the rf power source appears real, i.e. that the phase of the if voltage wave at the driving point is $\pi$ radians out of phase with the supply if current. This results in optimum operation of the if power source by minimizing the reflected power or dissipation in the power source. There are circumstances where this practice may not result in the best interaction between the if source and the beam dynamics. The required detuning may cause the accelerating cavity impedances presented to the adjacent upper and lower rotation bands to be sufficiently unbalanced so as to excite mode 1 coupled bunch instability. Also, in conditions of a ring partially filled with beam, the phase shift resulting from the power source drive current in the absence of beam can cause a bucket-beam mismatch for the beam following a gap, resulting in longitudinal emittance dilution. For these reasons it may be preferable to operate the accelerating cavities tuned exactly to resonance or detuned only a fraction of the amount necessary to cause the load to appear real. In such cases the phase of the If voltage at the driving point will vary from the $\pi$ radian condition. The required source current for a given load power (at constant if voltage) must then be increased by a factor sec $\delta$, where $\delta$ is the phase deviation from $\pi$ radians. This will result in an increase in the source dissipation and a corresponding decrease in the effective source resistance. These effects may sometimes be beneficial but they may also result in limitation of the available beam acceleration power. The size of these effects as a function of the phase shift $\delta$ is analysed in this section.

In Fig. B1(a) the anode current is shown as a function of the conduction angle $\Omega$ (or $\omega t$ ) in a cathode driven Y567B $150 \mathrm{~kW}$ tetrode for a real load condition. The control grid is grounded for $\mathrm{rf}$ but the dc level is adjustable. The dc anode voltage $\mathrm{V}_{\mathrm{a}}$ is set at $19.5 \mathrm{kV}$ and the anode if swing $V_{r f}$ is $17 \mathrm{kV}$, (i.e. the anode voltage swings down to $+2.5 \mathrm{kV}$ and $\mathrm{V}_{\mathrm{rf}}=0.872 \mathrm{~V}_{\mathrm{a}}$ ). The cathode drive level and the control grid dc bias are adjusted for peak current $\mathrm{L}_{\mathrm{p}}=45 \mathrm{~A}$, and for conduction over $\pi$ radians (class $B$ operation). The current pulse is well matched over the conduction angle by the function $I(\Omega)=\mathrm{I}_{\mathrm{p}} \cos ^{1.8} \Omega$. The anode current $\mathrm{I}_{\mathrm{av}}$, averaged over one if period, (equivalent to the $\mathrm{dc}$ anode current) is

$$
I_{a v}=\frac{I_{p}}{2 \pi} \int_{-\pi / 2}^{\pi / 2} \cos ^{1.8} \Omega d \Omega=0.260 I_{p}=11.7 \mathrm{~A} .
$$

In this case the fundamental Fourier component of $\mathrm{rf}$ current $\mathrm{I}_{\mathrm{rf}}=\mathrm{I}_{\mathrm{av}} \mathrm{F}_{1}(\Omega)$ is $1.67 \mathrm{I}_{\mathrm{av}}=19.5 \mathrm{~A}$. 
$F_{1}(\Omega)$ is obtained from a FFT of the current matching function. The if output power to the load is $P_{\text {out }}=1 / 2 V_{\text {rf }} \mathrm{I}_{\mathrm{rf}}=1 / 2(0.872) \mathrm{V}_{\mathrm{a}}(1.67) \mathrm{I}_{\mathrm{av}}=0.728 \mathrm{~V}_{\mathrm{a}} \mathrm{I}_{\mathrm{av}}=0.728 \mathrm{P}_{\mathrm{in}}$. The anode efficiency is near $73 \%$, reasonable for class $\mathrm{B}$ operation.

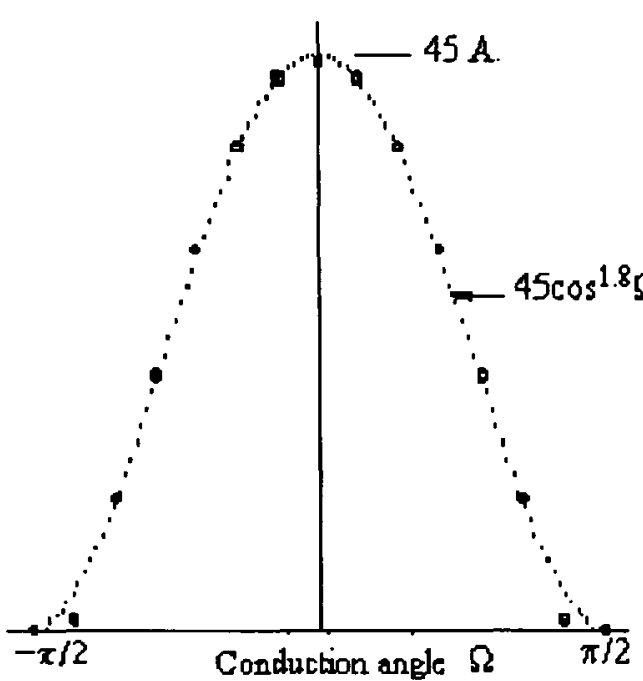

Figure 1B. Circles are anode current into real load. Dotted curve is $45 \cos 18 \Omega$.

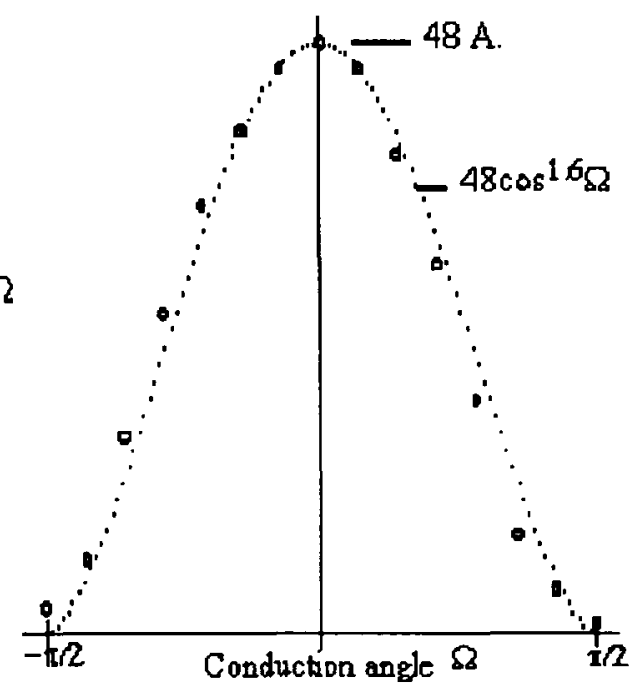

Figure 2B. Circles are anode current with anode voltage shitted in phase I radians with respect to carhode drive phase. Dotted curge is $48 \cos ^{1.6} \Omega$.

In Fig. B1(b) the tube anode current is shown for the same condition of cathode drive, grid bias, and anode supply voltage, but with the $17 \mathrm{kV}$ anode if voltage wave shifted in phase by $\delta=\pi / 4$ radians. Because the maximum drive point occurs at a higher anode voltage the peak current is increased to 48A. The current wave spans $\pi$ radians and it is sufficiently symmetric so that it is again well represented by a (slightly modified) cosine power law function $\mathrm{I}_{\mathrm{p}} \cos ^{1.6} \Omega$. There is apparently about a two degree shift in the phase of the current wave with respect to the cathode drive phase. The average anode current $I_{a v}$ is $0.272 I_{p}=13 \mathrm{~A}$. The fundamental Fourier component $F_{1}(\Omega)$ is $1.65 \mathrm{I}_{\mathrm{av}}=21.45 \mathrm{~A}$. As noted above, the $\mathrm{ff}$ current must be increased (probably by anode voltage sensing feedback) by a factor $\sec (\pi / 4)=1.414$ in order to deliver the required if power to the load. The power is $\mathrm{P}_{\text {out }}=1 / 2 \mathrm{~V}_{\mathrm{a}} \mathrm{I}_{\mathrm{rf}} \cos (\pi / 4)=0.508 \mathrm{~V}_{\mathrm{a}} \mathrm{I}_{\mathrm{av}}=0.508 \mathrm{P}_{\text {in }}$. The anode efficiency is decreased to near $51 \%$. 
The anode dissipation, normalized to constant power output, can be expressed,

$$
\frac{P_{\text {diss }}}{P_{\text {out }}}=\frac{V_{a} I_{p} \int_{-\pi / 2}^{\pi / 2} \cos m(\delta)(\Omega)\left[1-\frac{V_{r f}}{V_{a}} \cos (\Omega+\delta)\right] d \Omega}{\pi V_{r r_{r}} \cos (\delta)} .
$$

The exponent $m(\delta)$ scales with $\delta$ as $(1.8-0.255 \delta)$. By substituting $\mathrm{V}_{\mathrm{rf}}=(0.875) \mathrm{V}_{\mathrm{a}}$,

$$
f_{f}=F_{1}(\delta) I_{a v}=F_{1}(\delta) \frac{I_{p}}{2 \pi} \int_{-\pi / 2}^{\pi / 2} \cos ^{m \delta} \mathrm{Q} d \Omega \text {, and introducing a normalizing constant } \mathrm{N} \text { such that }
$$

the dissipation to output power ratio is unity for $\delta=0$, Eq. B2 can be written:

$$
\frac{P_{\text {diss }}}{P_{\text {out }}}=\frac{N}{F_{1}(\delta) \cos (\delta)}\left[1-\frac{0.872 \int_{-\pi / 2}^{\pi / 2} \cos ^{m(\delta)}(\Omega) \cos (\Omega-\delta) d \Omega}{\int_{-\pi / 2}^{\pi / 2} \cos ^{m(\delta)}(\Omega) d \Omega}\right] .
$$

This expression has been calculated for ten values of $\delta$ ranging from 0 to $\pi / 4$. The results along with the inverse of each point are plotted in Fig. B2. The rising curve represents the increase in anode dissipation over the value which obtains at $\delta=0$ for the same if power output and anode if voltage. The falling curve represents the reduction in effective $r_{p}$ resulting from the increase in source dissipation as it would affect cavity $Q$ and time constant. The dotted curves represent the function $(2-\cos (1.27 \delta))^{ \pm 2.5}$ which match the data reasonably well and may be used for ease of calculation. For $\delta=\pi / 4$ the anode dissipation is increased by a factor of 2.6 .

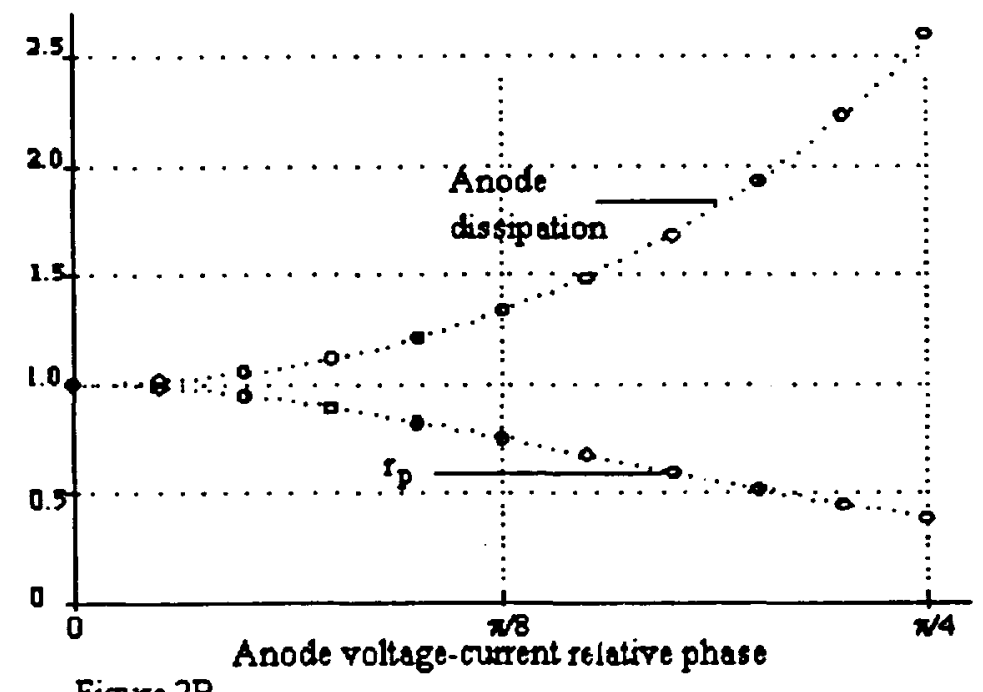

Fipure 2B 